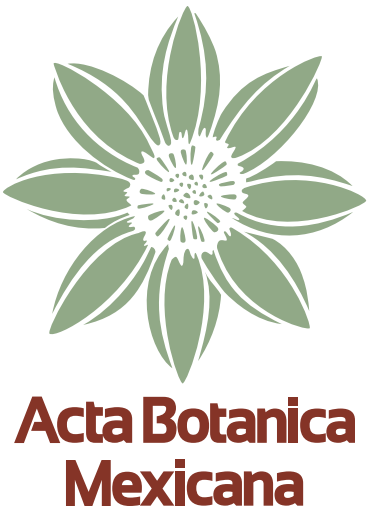

\title{
Confirmación taxonómica de Gelidium americanum (Gelidiaceae, Rhodophyta) en Tabasco, México, usando un enfoque morfológico y molecular
}

\author{
Taxonomic confirmation of Gelidium americanum \\ (Gelidiaceae, Rhodophyta) in Tabasco, Mexico, using a \\ morphological and molecular approach
}

\author{
Nataly Quiroz-González',3 (D), Ma. Guadalupe Rivas Acuña² (D), Ma. Edith Ponce Márquez' (D)
}

\begin{abstract}
Resumen:
Antecedentes y Objetivos: Gelidium americanum se distribuye ampliamente en la costa Atlántica de México; sin embargo, hasta ahora no se han realizado estudios que confirmen su identidad taxonómica utilizando marcadores moleculares y caracteres morfológicos. El objetivo del presente trabajo fue confirmar la identidad taxonómica con un enfoque morfológico y molecular, de los especímenes identificados como $G$. americanum recolectados previamente en la laguna costera de Mecoacán, Tabasco, México.

Métodos: Se recolectaron tres especímenes de G. americanum en la laguna Mecoacán, Tabasco, México. El material se analizó morfológicamente, mediante observaciones en un microscopio estereoscópico y microscopio óptico. Para el análisis molecular se extrajo ADN de los talos muestreados empleando el método CTAB; se amplificaron los marcadores rbcL y COI-5P con el kit Phire Plant Direct (PCR), las secuencias fueron editadas en Bioedit y alineadas en Clustal W. Enseguida se hizo un análisis de Máxima Verosimilitud en RaxML, otro de Inferencia Bayesiana en MrBayes y se calcularon las distancias genéticas en MEGA.

Resultados clave: Las secuencias obtenidas en el presente estudio para los marcadores rbcL y COI-5P se anidaron en el clado de las muestras identificadas previamente con marcadores moleculares como Gelidium americanum. Además, las distancias genéticas fueron mínimas, los caracteres morfológicos coincidieron con lo descrito previamente para otras localidades del Atlántico, a pesar de contar con un limitado número de especímenes. Conclusiones: Utilizando marcadores moleculares y caracteres morfológicos, se confirma la presencia de Gelidium americanum en la laguna Mecoacán a pesar de presentar características ambientales diferentes a las que habitualmente se han descrito para esta especie.
\end{abstract}

Palabras clave: algas rojas, COI-5P, filogenia, Golfo de México, morfología, rbcL.

\section{Abstract:}

Background and Aims: Gelidium americanum is widely distributed on the Atlantic coast of Mexico; however, so far no studies have been conducted to confirm its taxonomic identity using molecular markers and morphological characters. The objective of this study was to confirm the taxonomic identity, with a morphological and molecular approach, of the specimens identified as G. americanum previously collected in the coastal lagoon of Mecoacán, Tabasco, Mexico.

Methods: Three specimens of G. americanum were collected in the Mecoacán lagoon, Tabasco, Mexico. The material was morphologically analyzed by observations with stereoscopic and optical microscopes. For molecular analysis, DNA was extracted from the samples using the CTAB method; $r b c L$ and $C O I-5 P$ markers were amplified with the Phire Plant Direct kit (PCR), the sequences were edited in Bioedit and aligned in Clustal W. Next, a Maximum Verisimilitude analysis was done in RaxML, a Bayesian Inference analysis in MrBayes, and genetic distances were calculated in MEGA.

Key results: The sequences obtained in the present study for $r b c \mathrm{~L}$ and $\mathrm{COI-5P}$ markers were nested in the clade of samples previously identified with molecular markers as Gelidium americanum. In addition, genetic distances were minimal, the morphological characters coincided with what was previously described for other locations in the Atlantic, despite having a limited number of specimens.

Conclusions: Using molecular markers and morphological characters, the presence of Gelidium americanum in the Mecoacán lagoon is confirmed despite having different environmental characteristics to those that have usually been described for this species.

Key words: COI-5P, Gulf of Mexico, morphology, phylogeny, $r b c \mathrm{~L}$, red algae.

${ }^{1}$ Facultad de Ciencias, Universidad Nacional Autónoma de México, Av. Universidad 3000, Circuito Exterior s/n, Delegación Coyoacán, Ciudad Universitaria, 04510 Cd Mx., México.

2Universidad Juárez Autónoma de Tabasco, División Académica de Ciencias Biológicas, $0.5 \mathrm{~km}$ carretera Cárdenas, 86000 Villahermosa, Tabasco, México.

${ }^{3}$ Autor para la correspondencia: natalyquirozgonzalez@gmail.com
Recibido: 4 de septiembre de 2021.

Revisado: 12 de octubre de 2021

Aceptado por Rosario Redonda-Martínez: 26 de noviembre de 2021.

Publicado Primero en línea: 8 de diciembre de 2021. Publicado: Acta Botanica Mexicana 128 (2021).

(c) (7) Este es un artículo de acceso abierto bajo la licencia Creative Commons 4.0 Atribución-No Comercial (CC BY-NC 4.0 Internacional).
Citar como: Quiroz-González, N., M. G. Rivas Acuña y M. E. Ponce Márquez. 2021. Confirmación taxonómica de Gelidium americanum (Gelidiaceae, Rhodophyta) en Tabasco, México, usando un enfoque morfológico y molecular. Acta Botanica Mexicana 128: e1966. DOI: https://doi.org/10.21829/abm128.2021.1966 


\section{Introducción}

Gelidium americanum (W.R. Taylor) Santelices es una especie de alga roja (Rhodophyta) incluida dentro del orden Gelidiales y la familia Gelidiaceae. Fue descrita por Taylor (1943) como Pterocladia americana W.R. Taylor, con distribución en el Atlántico oriental y cuya localidad tipo es Port Antonio, Jamaica. La combinación G. americanum fue propuesta por Santelices (1976). En el Atlántico mexicano se le ha encontrado en localidades de Veracruz, Campeche, Quintana Roo (Mateo-Cid y Mendoza-González, 1991; Robledo et al., 2003; Galicia-García y Morales-García, 2007; Mendoza-González et al., 2007; Godínez-Ortega et al., 2009) y Tabasco. En este último estado la han registrado Ramírez (1996), Mendoza-González et al. (2017) y Quiroz-González et al. (2018) de tres localidades distintas (laguna La Machona, laguna Mecoacán y playa Paraíso), pero estos reportes estuvieron basados en las características morfológicas de los especímenes, sin haber confirmado su identidad molecular.

El empleo de marcadores moleculares, principalmente de plástidos y mitocondrias ( $r b c \mathrm{~L}, \mathrm{COI}-5 \mathrm{P}$ ), ha clarificado la situación taxonómica de algas del orden Gelidiales (p. ej., Boo et al., 2016a, b; Jamas et al., 2017; Boo y Hughey, 2019; Brunelli et al., 2019a, b; Perrone et al., 2019; Boo y Kim, 2020; Quiroz-González et al., 2020). Sin embargo, estos marcadores no han sido aplicados a especies mexicanas, con excepción de lo recientemente publicado por Quiroz-González et al. (2020) para las costas del Pacífico tropical mexicano. En el caso de $G$. americanum, Perrone et al. (2019) secuenciaron material proveniente del holotipo después de obtener fragmentos cortos para los marcadores $r b c \mathrm{~L}$ y $\mathrm{COI}-5 \mathrm{P}$, confirmando su identidad taxonómica. Algunas muestras procedentes del Mediterráneo incluidas en su estudio se agruparon en el mismo clado donde se encuentran el holotipo y algunos especímenes brasileños.

El objetivo del presente trabajo fue confirmar la identidad taxonómica de los especímenes identificados como $G$. americanum recolectados previamente en la laguna costera de Mecoacán, Tabasco, México, con un enfoque morfológico y molecular.

\section{Materiales y Métodos}

\section{Área de estudio}

Este trabajo se desarrolló a partir de un estudio prospectivo ficológico en la laguna Mecoacán; cuerpo de agua que po- see una extensión de 5168 ha y se ubica en la llanura costera de la cuenca del río Grijalva en el suroccidente del Golfo de México, en el estado de Tabasco, México (García-Cubas et al., 1990; Domínguez et al., 2003). El clima que predomina es de tipo Aw, cálido-húmedo con lluvias en verano y temperatura media anual de $26^{\circ} \mathrm{C}$ (Hernández-Ojendi et al., 2020). Es una laguna somera (0.9-1.2 $\mathrm{m}$ de profundidad), que recibe la descarga de los ríos Seco, Cuxcuchapa y Escarbado permanentemente, con marcados incrementos en la temporada de lluvias, que abarca desde septiembre hasta febrero; algunas partes de la laguna están ocupadas por bancos de mejillón y ostión, el fondo restante se encuentra cubierto por substratos suaves sin vegetación (Contreras, 1985; García-Cubas et al., 1990; Domínguez et al., 2003). La laguna Mecoacán presenta fluctuaciones importantes de salinidad a lo largo del año, oscilan entre 5 y 29 ups; en la época de precipitaciones (agosto-octubre) los valores rondan los 5-19 ups, derivando en un ambiente estuarino durante dicha temporada (Domínguez et al., 2003; Campos- Campos et al., 2014). El sitio de muestreo está representado en un mapa (Fig. 1) que se elaboró utilizando el programa QGIS v. 2.18.28 (QGIS Development Team, 2009).

\section{Colecta de material y análisis morfológico}

Se recolectaron tres especímenes identificados morfológicamente como $G$. americanum, en un solo sitio en la zona sur de la laguna durante la época de lluvias. Las muestras se encontraron en una zona protegida por bloques de concreto donde el flujo de agua era escaso. Estas recibieron dos tratamientos: en el primero, secciones de cada talo se colocaron en silica gel para el análisis molecular, en el otro, los talos completos se preservaron en formaldehído $\left(\mathrm{CH}_{2} \mathrm{O}\right)$ al $4 \%$ en agua de mar. A partir de los ejemplares conservados en $\mathrm{CH}_{2} \mathrm{O}$ se elaboraron preparaciones semipermanentes que se tiñeron con cristal violeta, para fijarlas en gelatina glicerinada al $75 \%$. Las secciones transversales y longitudinales del talo se cortaron manualmente, bajo un microscopio estereoscópico Nikon C-LEDS (Nikon, Konan, Minato-ku, Tokio, Japón), utilizando una hoja de afeitar. Las observaciones anatómicas se realizaron en un microscopio óptico Olympus CX23 (Olympus America Inc., Center Valley, Pennsylvania, EUA). Las fotografías de las características morfológicas se obtuvieron con una cámara Canon Eos Rebel T3 (Canon Inc., Tokio, Japón). 

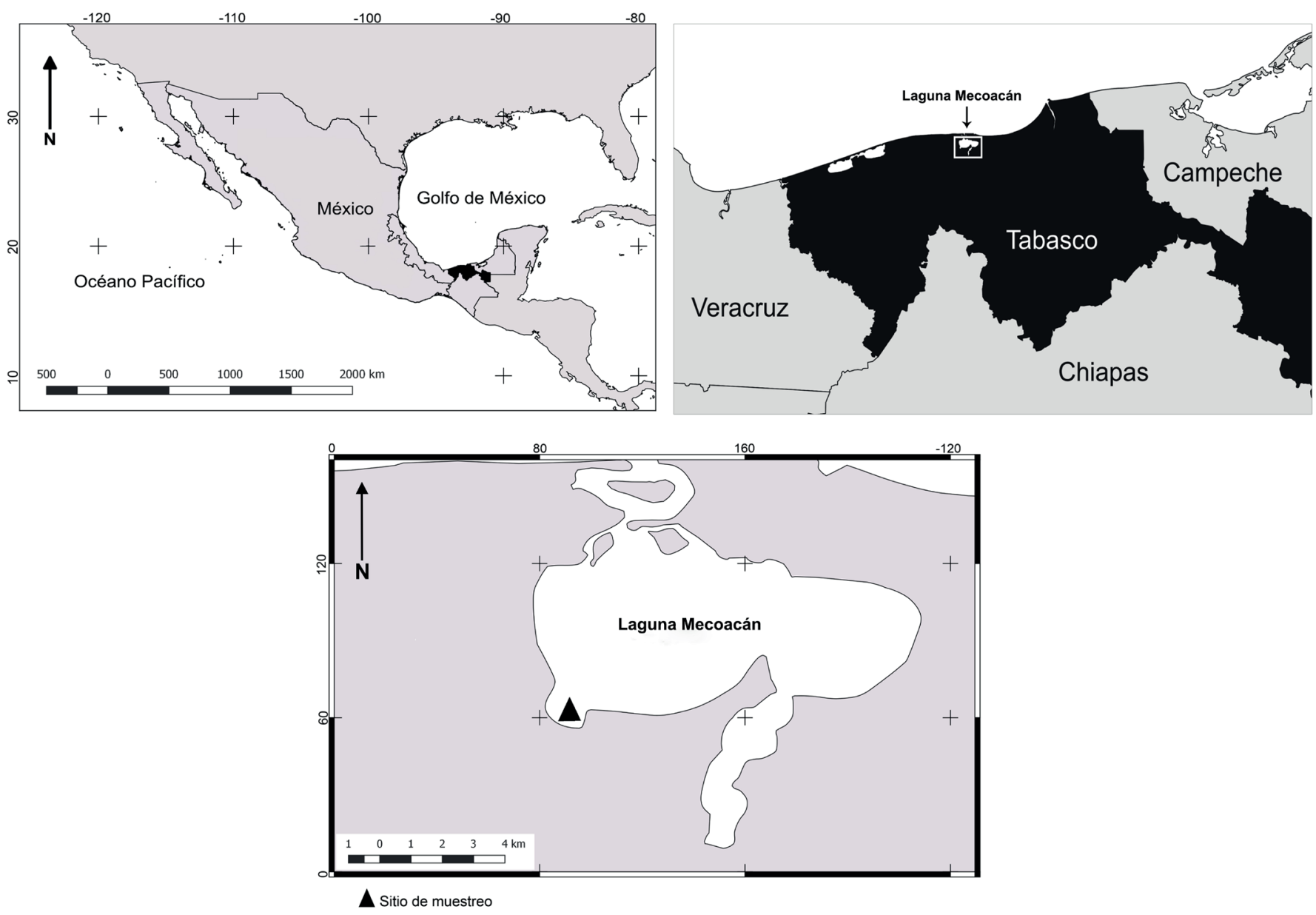

Figura 1: Ubicación de la laguna Mecoacán, Tabasco, México.

\section{Análisis filogenético}

La extracción de ADN de las muestras conservadas en silica gel se realizó utilizando el protocolo CTAB (Doyle y Doyle, 1990). Las reacciones de PCR se realizaron con el kit Thermoscientific Phire Plant Direct (C) (Thermo Fisher Scientific Inc., Waltham, MA, EUA). Los cebadores usados para la amplificación y secuenciación fueron: FrbcL-R753 y F57R1381 (Freshwater y Rueness, 1994) para rbcL, y GHalFGazR (Saunders, 2005) para COI-5P. La amplificación de los marcadores se hizo en un termociclador Techne Flexi-gene (Bibby Scientific, Maryland, EUA), siguiendo el protocolo de Freshwater y Rueness (1994). Los productos de PCR fueron secuenciados por Macrogen Inc. (Humanizing Genomas Macrogen, Seúl, Corea del Sur). Las secuencias fueron editadas con el programa Bioedit v. 7.0.5.3 (Hall, 1999), mientras que el alineamiento se llevó a cabo en Clustal W v. 2.0.3 (Thompson et al., 1994).
Los alineamientos de $r b c \mathrm{~L}$ consistieron en tres secuencias obtenidas en este trabajo y 27 de GenBank (2021); en el caso de COI-5P, tres son producto de este estudio y 28 adquiridas de GenBank (2021) (Cuadro 1). Ptilophora scalaramosa (Kraft) Norris, Gelidiophycus freshwateri Boo, Park \& Boo y Capreolia implexa Guiry \& Womersley fueron utilizados como grupos externos. Se empleó MEGA v. 7.0 (Kumar et al., 2016) para calcular distancias genéticas. EI cálculo del mejor modelo de evolución de secuencias para cada conjunto de datos se realizó con el programa JModelTest v. 3.7 utilizando el Criterio de Información Akaike (Dariba et al., 2012), siendo GTR + G + I el más adecuado para ambos conjuntos de datos. Las filogenias de las secuencias de $r b c \mathrm{~L}$ y $\mathrm{COI}-5 \mathrm{P}$ se infirieron utilizando Máxima verosimilitud (ML) e Inferencia Bayesiana (BI). El análisis de ML se realizó en RaxML v. 8.0.0X (Stamatakis, 2014), empleando el algoritmo de búsqueda heurística, se realizaron 
Cuadro 1: Secuencias de rbcL y COI-5P generadas en el presente estudio y obtenidas de GenBank (2021). Con asterisco se indican las accesiones de las secuencias obtenidas en este trabajo.

\begin{tabular}{|c|c|c|c|}
\hline \multirow{2}{*}{ Especies } & \multirow{2}{*}{ Localidad de muestreo } & \multicolumn{2}{|c|}{ Gen Bank } \\
\hline & & $r b c L$ & COI-5P \\
\hline \multirow[t]{6}{*}{ Gelidium americanum (W.R. Taylor) Santelices } & México: Mecoacán, Tabasco & *MW788675 & *MW760396 \\
\hline & & *MW788676 & *MW788673 \\
\hline & & *MW788677 & *MW788674 \\
\hline & Jamaica: Port Antonio & МК034311 & МК034308 \\
\hline & Brasil & KX555615 & KX574741 \\
\hline & Brasil & KX555614 & KX574740 \\
\hline \multirow[t]{4}{*}{ G. calidum Jamas, Iha \& Fujii } & Brasil: Pernambuco & KT208120 & KT208040 \\
\hline & Brasil & KX555612 & KT574739 \\
\hline & Brasil & - & KX574739 \\
\hline & & & KX574737 \\
\hline G. capense (S.G. Gmelin) P.C. Silva & Sudáfrica: Cape Peninsula, False Bay & L22461 & HM629858 \\
\hline G. caulacantheum J. Agardh & Nueva Zelanda: Porirua Harbor & U00103 & HM629860 \\
\hline $\begin{array}{l}\text { G. carolinianum C. Perrone, D.W. Freshwater, A. } \\
\text { Bottalico, G.H. Boo \& S.M. Boo }\end{array}$ & $\begin{array}{l}\text { Estados Unidos de América: North Carolina, } \\
\text { Topsail Sound, Pender Co. }\end{array}$ & MG272418 & MG800613 \\
\hline G. corneum (Hudson) J.V. Lamoroux & Marruecos & HM629821 & HM629861 \\
\hline G. crinale (Hare ex Turner) Gaillon & $\begin{array}{l}\text { Australia: Rottnest Island, Green Island / } \\
\text { Reino Unido: Sidmouth }\end{array}$ & HQ412493 & JX096542 \\
\hline G. elegans Kützing & Corea del Sur: Seogeochado, Jindo & HM629829 & HM629869 \\
\hline G. galapagense W.R. Taylor & Ecuador: Banks Bay, Isla Isabela, Galapagos & KX423476 & KX423473 \\
\hline G. gabrielsonii Hughey \& G.H. Boo & $\begin{array}{l}\text { Estados Unidos de América: California, } \\
\text { Asilomar, Monterey County }\end{array}$ & MH780748 & MG922859 \\
\hline G. hommersandii A.J.K. Millar \& D.W. Freshwater & Australia: New South Wales & JX891625 & JX891599 \\
\hline G. indonesianum K.M. Kim, G.S. Gerung \& S.M. Boo & Indonesia: Pameungpeuk beach, Java & JF330216 & JF330215 \\
\hline G. isabelae W.R. Taylor & $\begin{array}{l}\text { Ecuador: Pt. Albemarle, Isla Isabela, } \\
\text { Galapagos }\end{array}$ & KX423477 & KX427231 \\
\hline G. japonicum (Harvey) Okamura & Japón: Choshi, Chiba & HM629830 & HM629870 \\
\hline $\begin{array}{l}\text { G. jejuense K.M. Kim, I.K. Hwang, H.S. Yoon \& S.M. } \\
\text { Boo }\end{array}$ & Corea: Jongdal, Jeju & JQ340407 & JQ340445 \\
\hline G. kathyanniae G.H. Boo \& Hughey & $\begin{array}{l}\text { Estados Unidos de América: California, Point } \\
\text { Pinos, Pacific Grove, Monterey }\end{array}$ & MH780752 & MH780746 \\
\hline G. longipes Agardh & Nueva Zelanda & AY648021 & - \\
\hline G. pacificum Okamura & Japón: Marine Station, Chiba & HM629831 & HM629871 \\
\hline G. purpurascens N.L. Gardner & $\begin{array}{l}\text { Estados Unidos de América: California, San } \\
\text { Juan Island; California, Agate Beach }\end{array}$ & U00979 & HM629873 \\
\hline G. pusillum (Stackhouse) Le Jolis & Reino Unido: Sidmouth & U00999 & JX096551 \\
\hline G. sclerophyllum W.R. Taylor & Costa Rica: Guanacaste, Montezuma & KC192651 & KC288159 \\
\hline Capreolia implexa Guiry \& Womersley & $\begin{array}{l}\text { Nueva Zelanda: Ringaringa Beach, Stewart } \\
\text { Island }\end{array}$ & KM204080 & KM204044 \\
\hline $\begin{array}{l}\text { Gelidiophycus freshwateri G.H. Boo, J.K. Park \& S.M. } \\
\text { Boo }\end{array}$ & Corea: Daecheon, Boryeong & JX891603 & JX891571 \\
\hline Ptilophora scalaramosa (Kraft) R.E. Norris & Filipinas: Sorsogon Province, Bulusan & KT920275 & КT920401 \\
\hline
\end{tabular}


100 réplicas de secuencias aleatorias y posteriormente, 100 réplicas de bootstrap. El análisis Bayesiano se hizo con MrBayes v. 3.2.1 (Ronquist et al., 2012) usando cuatro cadenas de Markov Monte Carlo comenzando con un árbol al azar y predefiniendo un muestreo de los datos cada 200 generaciones durante 2,000,000 de generaciones, se utilizó un "burnin" de $25 \%$. Los árboles resultantes de los dos análisis fueron visualizados y editados en FigTree (Rambaut, 2010).

\section{Resultados}

Los especímenes analizados en el presente estudio coincidieron con la mayoría de las características morfológicas señaladas para G. americanum por otros autores (Cuadro 2). Las muestras se diferenciaron de los talos descritos en estudios previos por el intervalo en longitud y ancho, disposición de las células medulares y abundancia de los filamentos rizoidales internos.

\section{Taxonomía}

Gelidium americanum (W.R. Taylor) Santelices, Phycologia 15: 173. 1976. Fig. 2.

TIPO: JAMAICA. Port Antonio, VII.1990. C. E. Pease y E. Butler s.n. (MICH 1306116).

इ Pterocladia americana W.R. Taylor, Papers Michigan Acad. Sc. 28: 153. 1943.

Talos erectos con apariencia arbustiva, pardo-rojizos, 2-4 cm de altura, 1-2 mm de ancho; ramificación de segundo a tercer orden irregular en la parte inferior a alterna y subpinnada en la parte superior; talo comprimido a aplanado, ramas ligeramente atenuadas en la base; células medulares, 14-16.5 $\mu \mathrm{m}$ de diámetro, dispuestas en tres hileras, rodeadas de abundantes filamentos rizoidales; células corticales, 6.5-9.5 $\mu \mathrm{m}$ de diámetro, dispuestas en una hilera; estructuras reproductivas no observadas (Fig. 2).

Hábitat: creciendo sobre concreto y conchas de Crassostrea virginica (Gmelin, 1791). En una zona protegida, con muy poco movimiento, sitio sin exposición directa al sol.
Distribución: México: Campeche, Laguna de Términos (Ortega, 1995; Ortega et al., 2001; Mateo-Cid et al., 2013); Quintana Roo, Los Manchones, Puerto Morelos, Xcacel, San Juan Cozumel (Mateo-Cid y Mendoza-González, 1991; Mendoza-González y Mateo-Cid, 1992; Dreckmann et al., 1996; Ortega et al., 2001); Tabasco, laguna Mecoacán, La Machona, Playa Paraíso (Mendoza-González et al., 2017; Quiroz-González et al., 2018; este trabajo); Tamaulipas, La Carbonera San Fernando, Ciudad Madero, playa Lauro Villar, Matamoros (Ortega et al., 2001); Veracruz, Morro la Mancha (Ortega et al., 2001; De la Garza-Flores, 2003; Núñez-Reséndiz, 2009).

Material examinado: MÉXICO. Tabasco, laguna Mecoacán, 0 m, 6.IX.2017, N. Quiroz CA280 (UJAT), CA281 (UJAT), CA282 (UJAT 3).

\section{Análisis filogenético}

La filogenia molecular para el marcador $r b c \mathrm{~L}$ consistió en el análisis de 30 secuencias (tres generadas en este estudio) con 1200 pb (pares de bases), de las cuales 410 pb eran variables. Mientras que para COI-5P incluyó 31 secuencias (tres producto de este trabajo) con 515 pb, de las cuales 223 pb eran variables. Los análisis de Máxima Verosimilitud e Inferencia Bayesiana produjeron árboles con topologías similares con ambos marcadores. Se generaron cladogramas consenso para los dos (Figs. 3, 4). Las tres secuencias de rbcL y COI-5P formaron clados con alta probabilidad posterior y valores de soporte de bootstrap (1 y 100, respectivamente).

Para ambos árboles filogenéticos los especímenes analizados en el presente estudio se agruparon en el mismo clado que las muestras de $G$. americanum confirmadas por Perrone et al. (2019), incluyendo el holotipo procedente de Jamaica y muestras brasileñas, Se ubicaron como grupo hermano de G. calidum Jamas, Iha \& Fujii (Figs. 3, 4), especie descrita por Jamas et al. (2017) para Pernambuco, Brasil. Las distancias genéticas intraespecíficas de los especímenes de Tabasco fueron $0-0.01 \%$ para $r b c L$ y $0-0.02 \%$ en COI-5P. La distancia genética entre los especímenes de la laguna Mecoacán y los de G. americanum de GenBank fue de $0.6 \%$ con COI-5P y $0 \%$ para rbcL. Comparando con las muestras de $G$. calidum, la distancia fue $3.2-3.4 \%$ con $C O I-5 P$ y $0.9 \%$ en el caso de rbcL. Con el resto de las especies de Gelidium los 
Cuadro 2: Comparación de los caracteres morfológicos descritos por otros autores y en el presente estudio para Gelidium americanum (W.R. Taylor) Santelices.

\begin{tabular}{|c|c|c|}
\hline Caracteres & $\begin{array}{l}\text { Taylor (1943), Santelices (1976); Perrone et al. } \\
\text { (2019) }\end{array}$ & Este trabajo \\
\hline Hábito & Erecto, parecido a un arbusto & Erecto, parecido a un arbusto \\
\hline Forma del talo & $\begin{array}{l}\text { Comprimido a aplanado, espatulado a } \\
\text { lanceolado; ramas ligeramente atenuadas en } \\
\text { la base }\end{array}$ & $\begin{array}{l}\text { Comprimido a aplanado, ramas ligeramente atenuadas en la } \\
\text { base }\end{array}$ \\
\hline Patrón de ramificación & $\begin{array}{l}\text { Irregular en la parte inferior, alterna a } \\
\text { subpinnada en el tercio superior; sin un eje } \\
\text { central dominante }\end{array}$ & $\begin{array}{l}\text { Irregular en la parte inferior a alterna en la parte superior; sin } \\
\text { un eje central dominante }\end{array}$ \\
\hline Órdenes de ramificación & $2-4$ & $2-3$ \\
\hline Longitud & $0.5-6 \mathrm{~cm}$ & $2-4 \mathrm{~cm}$ \\
\hline Ancho & $0.4-2 \mathrm{~mm}$ & $1-2 \mathrm{~mm}$ \\
\hline Células medulares & Sin orden evidente & En tres hileras \\
\hline Filamentos rizoidales internos & Escasos y dispersos en la médula & Abundantes en la médula \\
\hline
\end{tabular}
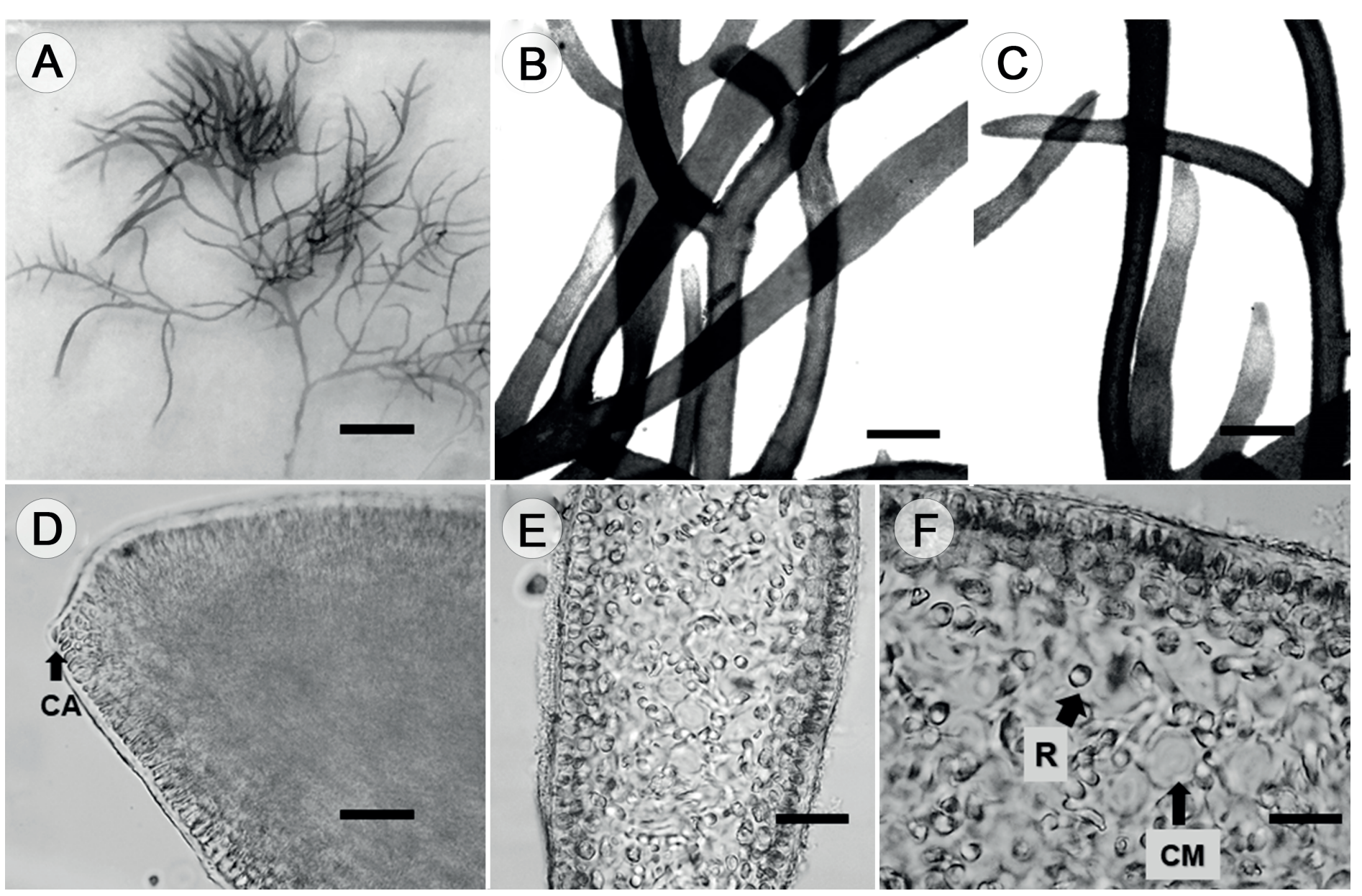

Figura 2: Características vegetativas de Gelidium americanum (W.R. Taylor) Santelices. A. talo completo, N. Quiroz CA280 (UJAT); B-C. detalle del talo. D. célula apical $(C A)$. Escala $=100 \mu \mathrm{m}$. E. sección transversal. F. sección transversal, con células medulares indicadas $(C M)$ y rizinas $(R)$. Escalas; $A=0.2$ $\mathrm{mm} ; \mathrm{B}=100 \mu \mathrm{m} ; \mathrm{C}=100 \mu \mathrm{m} ; \mathrm{D}=100 \mu \mathrm{m} ; \mathrm{E}=40 \mu \mathrm{m} ; \mathrm{F}=25 \mu \mathrm{m}$. 
valores oscilaron entre 5.8 y $13.6 \%$ en $C O I-5 P$ y $1.1-10 \%$ en rbcL.

\section{Discusión}

En el presente trabajo se confirma taxonómicamente la identidad de tres especímenes identificados previamente como G. americanum para las costas de Tabasco, México, empleando herramientas morfológicas y moleculares. Los especímenes analizados en este estudio presentan pocas diferencias con respecto a los caracteres morfológicos de los talos descritos por otros autores (Taylor, 1943; Santelices, 1976; Perrone et al., 2019). Las principales corresponden a los intervalos en longitud y ancho de los talos: en los ejemplares de Gelidium americanum estudiados tienen de 2-4 cm de longitud y 1-2 mm de ancho, mientras que en trabajos previos fueron de $0.5-6 \mathrm{~cm}$ de longitud y $0.4-2 \mathrm{~mm}$ de ancho (Taylor, 1943; Santelices, 1976; Perrone et al., 2019).
La distribución de las células medulares y la cantidad de filamentos rizoidales internos es otra diferencia; también el arreglo de las células medulares. En publicaciones previas se registraron células medulares sin un arreglo aparente, mientras que en el presente trabajo se observaron dispuestas en tres hileras. Además, los filamentos rizoidales internos se han reportado como escasos alrededor de la médula (Taylor, 1943; Perrone et al., 2019), pero en este estudio realizado fueron abundantes. Las diferencias morfológicas observadas en estos especímenes con respecto a las descritas anteriormente (Taylor, 1943; Santelices, 1976; Perrone et al., 2019) podrían relacionarse con la presencia de G. americanum en un ambiente con características distintas a aquellas en las que habitualmente se encuentra.

Con el presente estudio, además de confirmar la identidad taxonómica de los especímenes recolectados, que se ubicaron en el mismo clado que el holotipo de G. ameri-

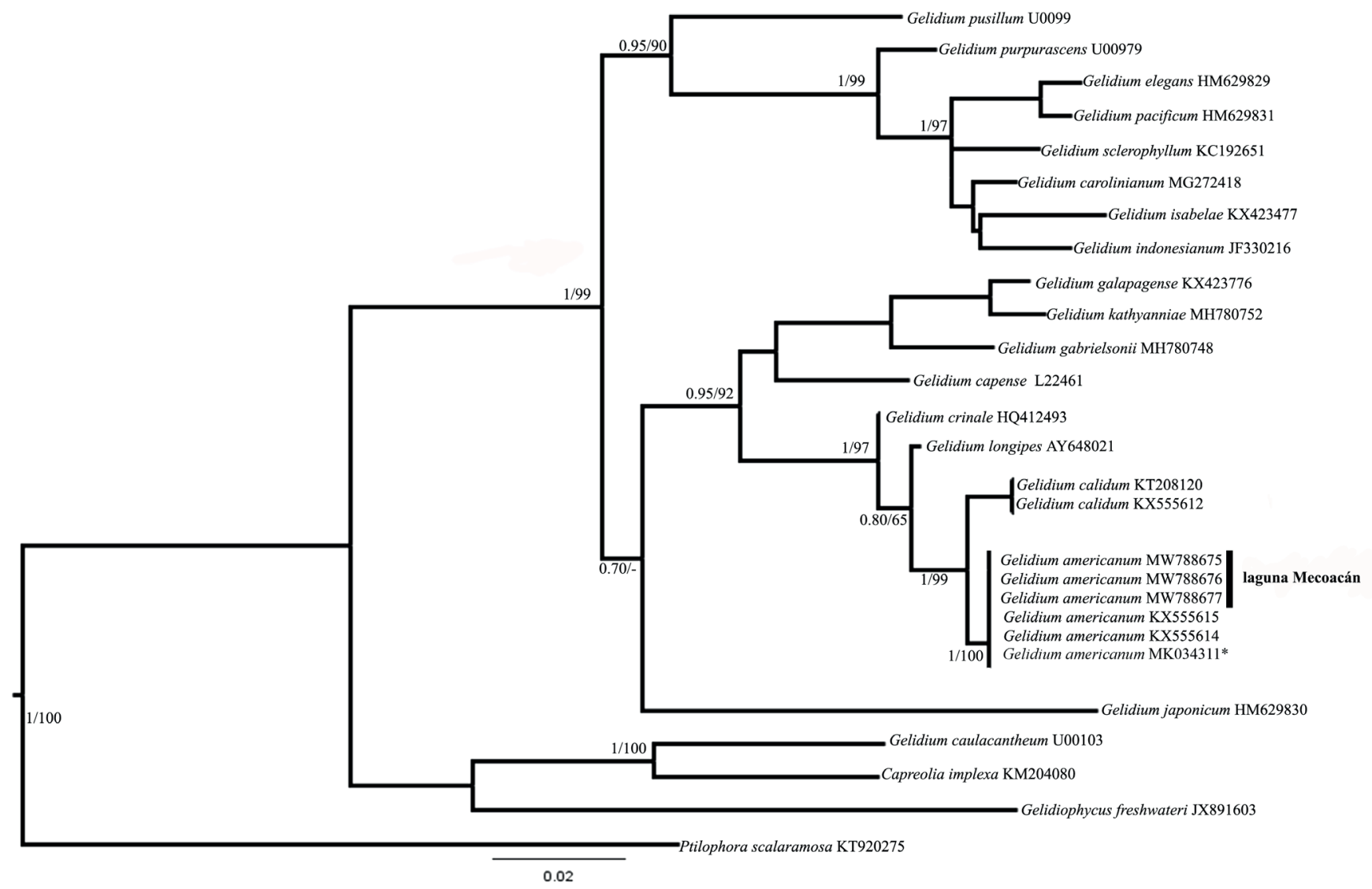

Figura 3: Árbol consenso inferido a partir de secuencias de rbcL para Gelidium J.V. Lamouroux. Los valores de probabilidad posterior y bootstrap se muestran en las ramas. *Holotipo de G. americanum (W.R. Taylor) Santelices. 
canum, es posible demostrar su cercanía filogenética con $G$. crinale (Hare ex Turner) Gaillon y G. calidum tal como señalaron Perrone et al. (2019). Tanto G. calidum, como G. crinale se distribuyen, al igual que G. americanum, en el océano Atlántico (Jamas et al., 2017; Perrone et al., 2019) y hasta el momento únicamente los últimos dos taxones se han registrado en las costas del Golfo de México y Caribe mexicano. Gelidium calidum es una especie descrita recientemente, cuya distribución está restringida a Brasil (Jamas et al., 2017), no se descarta que se encuentre en el Atlántico mexicano, por lo que era de suma importancia conocer la relación filogenética entre los ejemplares analizados y G. calidum.
Los valores de divergencias de nucleótidos para ambos marcadores a nivel intra e interespecífico coinciden con los indicados previamente en Gelidiales y otras algas rojas (Boo et al., 2016a, b; Jamas et al., 2017; Boo y Hughey, 2019; Brunelli et al., 2019a, b; Perrone et al., 2019; Boo y Kim, 2020; Quiroz-González et al., 2020).

En el Golfo de México y Mar Caribe G. americanum ha sido registrada con frecuencia en localidades litorales, intermareales y lagunas costeras (Ramírez, 1996; Mendoza-González et al., 2017; Quiroz-González et al., 2018). Sin embargo, su condición tolerante a cambios de salinidad no se ha reconocido abiertamente como en otras especies de

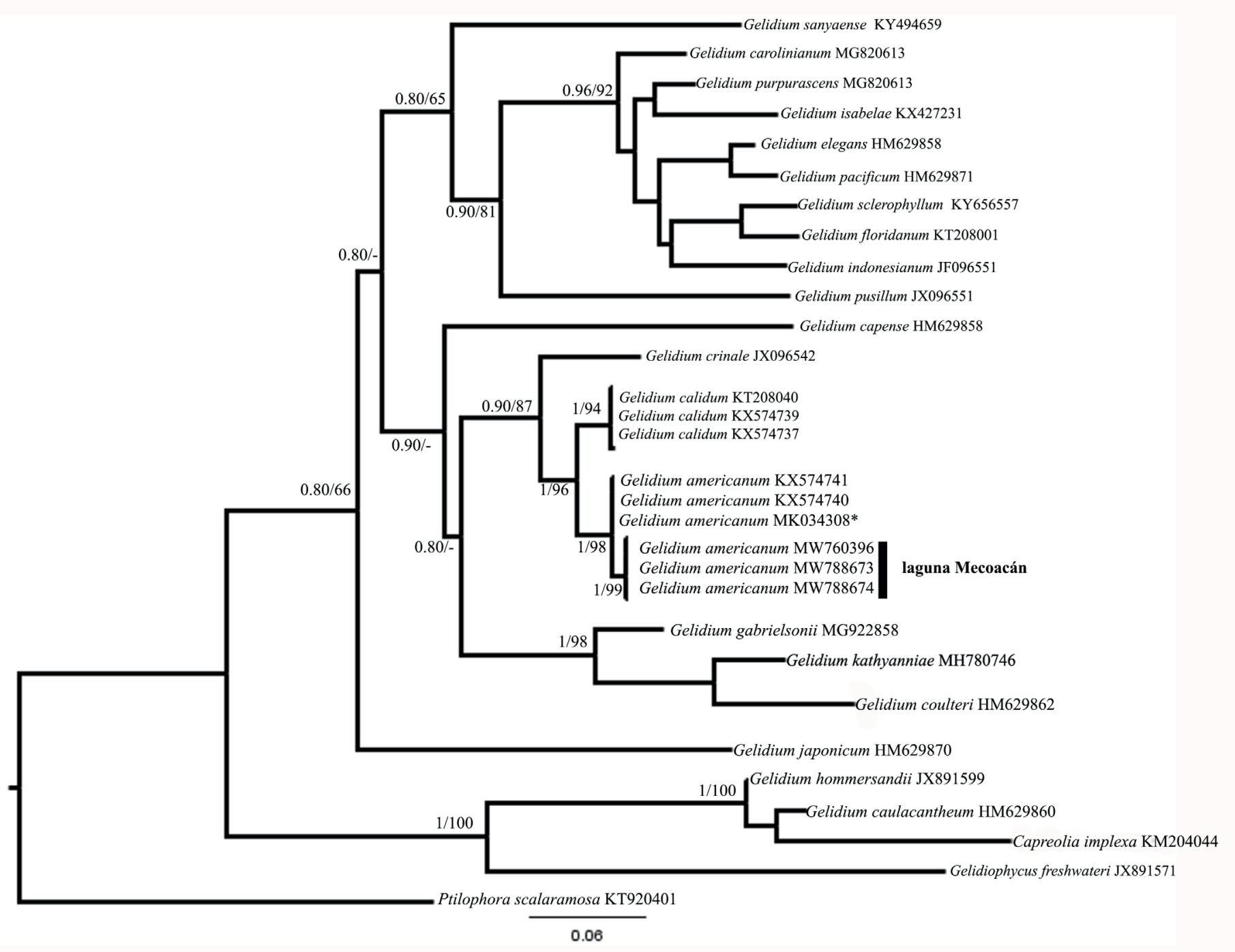

Figura 4: Árbol consenso inferido a partir de secuencias de COI-5P para Gelidium J.V. Lamouroux. Los valores de probabilidad posterior y bootstrap se muestran en las ramas. *Holotipo de G. americanum (W.R. Taylor) Santelices. 
Gelidium: G. amansii (J.V. Lamouroux) J.V. Lamouroux, G. coulteri Harvey y G. floridanum W.R. Taylor. En ellas se ha evaluado el impacto de los cambios de salinidad en su fisiología y reproducción (Macler, 1988; Fralick et al., 1990; Filipin et al., 2016). La presencia de especímenes de G. americanum en época de lluvias en la laguna Mecoacán podría sugerir que es una especie eurihalina, ya que está sometida a cambios drásticos en los valores de salinidad a largo del año, fluctuaciones que han sido previamente registradas en este sitio (Domínguez et al., 2003). Por lo anterior, es importante plantear estudios futuros que evalúen el impacto real de los cambios de salinidad en la laguna Mecoacán para G. americanum. Además, las especies del género Gelidium se encuentran por lo general en sustrato rocoso, a menudo en costras coralinas, características ecológicas distintas a las que se observaron en el sitio donde se recolectaron los especímenes analizados, ya que estaban adheridos a bloques de concreto, sobre conchas de Crassostrea virginica y a escasa profundidad.

Otro factor que es muy importante para el desarrollo y crecimiento de Gelidium es el hidrodinamismo, pues los miembros de este género han sido asociados con movimiento rápido del agua y se han registrado en sitios expuestos, con cambios importantes en la marea (Santelices, 1988; 1991). Algunas excepciones son los especímenes localizados en zonas templadas, donde especies como G. pusillum (Stackhouse) Le Jolis y G. spinosum (S.G. Gmelin) P.C. Silva se han encontrado en sitios fangosos, protegidos y con movimiento de agua muy ligero (Santelices, 1988). Las muestras analizadas en el presente trabajo se encontraron en zonas sin flujo importante de agua, en sitios protegidos, lo que coincide con lo señalado anteriormente, pero a diferencia de lo que se indica para otras especies, las de este estudio están en una zona con clima tropical. La variación en talla con respecto a otros registros podría deberse a la falta de flujo en el agua, ya que como han señalado otros autores (Anderson y Charters, 1982; Pacheco-Ruíz y Zertuche-González, 1995), este factor influye directamente en el crecimiento del talo de Gelidium.

A pesar de encontrarse en condiciones ambientales distintas a las que se describen comúnmente para el crecimiento de Gelidium, es posible confirmar la presencia de $G$. americanum en la laguna Mecoacán, Tabasco, México, gracias a las evidencias morfológicas y moleculares aquí pre- sentadas. Este trabajo contribuye además con las primeras secuencias de esta especie provenientes de material de poblaciones mexicanas. Resulta fundamental seguir trabajando en la identificación molecular y morfológica de especies mexicanas de Gelidium, sobre todo en ambientes tan particulares como las lagunas costeras.

\section{Contribución de autores}

MRA y NQG Ilevaron a cabo las recolecciones del material en el área de muestreo. NQG y MRA realizaron la revisión del material muestreado, la elaboración de preparaciones, la toma de medidas, y la identificación y descripción de los ejemplares. NQG y MPM Ilevaron a cabo el análisis molecular. NQG, MRA y MPM escribieron el manuscrito. Todos los autores contribuyeron a la discusión, revisión y aprobación del manuscrito final.

\section{Agradecimientos}

Al Herbario de la Universidad Juárez Autónoma de Tabasco por las facilidades para la recolecta y el procesamiento del material. Al laboratorio de Ficología (Biodiversidad Marina) de la Universidad Nacional Autónoma de México por el préstamo de los equipos e instalaciones. A Dení Rodríguez por sus valiosos comentarios.

\section{Financiamiento}

El presente trabajo no contó con financiamiento externo, los recursos fueron aportados por los autores.

\section{Literatura citada}

Anderson, S. M. y A. C. Charters. 1982. A fluid dynamics study of seawater flow through Gelidium nudifrons. Limnology and Oceanography 27(3): 399-412. DOI: https://doi.org/10.4319/ lo.1982.27.3.0399

Boo, G. H. y J. R. Hughey. 2019. Phylogenomics and multigene phylogenies decipher two new cryptic marine algae from California, Gelidium gabrielsonii and Gelidium kathyanniae (Gelidiales, Rhodophyta). Journal of Phycology 55(1): 160172. DOI: https://doi.org/10.1111/jpy.12802

Boo, G. H. y K. M. Kim. 2020. A new species of marine algae from Korea based on morphology and molecular data: Gelidium palmatum sp. nov. (Gelidiales, Rhodophyta). Algae 35(1): 3343. DOI: https://doi.org/10.4490/algae.2020.35.3.6 
Boo, G. H., J. R. Hughey, K. A. Millery S. M. Boo. 2016a. Mitogenomes from type specimens, a genotyping tool for morphologically simple species: ten genomes of agar-producing red algae. Scientific Reports 6: 35337. DOI: https://doi.org/10.1038/ srep35337

Boo, G. H., L. Le Gall, K. A. Miller, D. W. Freshwater, T. Wernberg, R. Terada, K. J. Yoon y S. M. Boo. 2016b. A novel phylogeny of the Gelidiales (Rhodophyta) based on five genes including the nuclear CesA, with descriptions of Orthogonacladia gen. nov. and Orthogonacladiaceae fam. nov. Molecular Phylogenetics and Evolution 101: 359-372. DOI: https://doi.org/10.1016/j. ympev.2016.05.018

Brunelli, B., D. Milstein, S. M. Boo y M. T. Fujii. 2019a. Gelidium guimaraesiae sp. nov. (Gelidiaceae, Rhodophyta) from the Western Atlantic segregated from $G$. floridanum by morphological and molecular evidence. Phytotaxa 388 (4): 275-286. DOI: https://doi.org/10.11646/phytotaxa.388.4.3

Brunelli, B., M. Jamas, D. Milstein, S. M. Boo y M. T. Fujii. 2019b. Gelidium brasiliense sp. nov. (Gelidiales, Rhodophyta): a diminutive agarophyte from Brazil. Journal of Applied Phycology 31(2): 951-958. DOI: https://doi.org/10.1007/ s10811-018-1678-9

Campos-Campos, B., T. N. Custodio-Osorio, C. Torres-Sauret, M. G. Rivas-Acuña y L. Cruz Rosado. 2014. Registro preliminar de la composición fitoplanctónica de la laguna Mecoacán, Paraíso, Tabasco, México. Kuxulkab'18(34): 65-72.

Contreras, E. F. 1985. Las lagunas costeras mexicanas. Centro de Ecodesarrollo-Secretaría de Pesca. Cd. Mx., México. 253 pp.

Dariba, D., G. L. Taboada, R. Doallo y D. Posada. 2012. jModel Test 2: more models, new heuristics and parallel computing. Nature Methods 9(8): 772. DOI: https://doi.org/10.1038/ nmeth.2109

De la Garza-Flores, C. 2003. Clave genérica de las algas rojas marinas macroscópicas y comunes de las costas de Veracruz, México. Tesis de licenciatura. Facultad de Estudios Superiores Iztacala, Universidad Nacional Autónoma de México. Cd. Mx., México. 79 pp.

Domínguez, J. C., A. Sánchez, R. Floridoy E. Barba. 2003. Distribución de macrocrustáceos en laguna Mecoacán, al sur del Golfo de México. Hidrobiología 13(2): 127-136.

Doyle, J. J. y J. L. Doyle. 1990. Isolation of plant DNA from fresh tissue. Focus 12(1): 13-15.
Dreckmann, K. M., I. Stout y A. S. Granados. 1996. Lista actualizada de las algas marinas bentónicas de Puerto Morelos, Quintana Roo, Caribe Mexicano. Polibotánica 3: 1-17.

Filipin, E. P., Z. L. Bouzon., L. Ouriques, L., D. T. Perreira, F. Braum, É. C. Schmidt y C. Simioni. 2016. Evaluation of salinity effects on the release, adhesion, and germination of the tetraspores of Gelidium floridanum (Rhodophyta, Florideophyceae). Journal of Applied Phycology 28(5): 2925-2938. DOI: https:// doi.org/10.1007/s10811-016-0836-1

Fralick, R. A., H. P. Baldwin, A. I. Neto y E. J. Hehre. 1990. Physiological responses of Pterocladia and Gelidium (Gelidiales, Rhodophyta) from the Azores, Portugal. Hydrobiologia 204(1): 479-482. DOI: https://doi.org/10.1007/BF00040274

Freshwater, D. W. y J. Rueness. 1994. Phylogenetic relationships of some European Gelidium (Gelidiales, Rhodophyta) species, based on $r b c L$ nucleotide sequence analysis. Phycologia 33(3): 187-194. DOI: https://doi.org/10.2216/i0031-888433-3-187.1

Galicia-García, C. y A. Morales-García. 2007. Investigaciones sobre macroalgas realizadas en el sistema arrecifal veracruzano. In: Granados Barba, A., L. G. Abarca Arenas y J. M. Vargas Hernández (eds.). Investigaciones Científicas en el Sistema Arrecifal Veracruzano. Universidad Autónoma de Campeche. Campeche, México. 304 pp.

García-Cubas, A., D. F. Escobar, A. I. González y M. Reguero. 1990. Moluscos de la laguna Mecoacán, Tabasco, México: Sistemática y Ecología. Anales del Instituto de Ciencias del Mar y Limnología, Universidad Nacional Autónoma de México 17: 1-30.

Genbank. 2021. National Center for Biotechnology Information http://www.ncbi.nlm.nih.gov/genbank/ (consultado julio de 2021).

Godínez-Ortega, J. L., P. Ramírez-García y K. Pedraza-Venegas. 2009. Cambios en la flora béntica de Arrecife Hornos (Veracruz, México). TIP Revista Especializada en Ciencias Químico-Biológicas 12(2): 59-65.

Hall, T. A. 1999. BioEdit: a user-friendly biological sequence alignment editor and analysis program for Windows 95/98/ NT. Nucleic Acids Symposium Series 41: 95-98.

Hernández-Ojendi, R., L. A. Ayala-Pérez, A. Esquivel-Herrera y B. I. Vega-Rodríguez. 2020. Estructura de la comunidad de peces de la laguna Mecoacán, Tabasco, México. JAINA Costas y 
Mares ante el Cambio Climático 2(1): 1-18. DOI: https://doi. org/10.26359/52462.0120

Jamas, M., C. Iha, M. C. Oliveira, S. M. P. B Guimarães y M. T. Fujii. 2017. Morphological and molecular studies on Gelidiaceae and Gelidiellaceae (Gelidiales, Rhodophyta) from Brazil with description of the new species Gelidium calidum. Phytotaxa 314(2): 195-218. DOI: https://doi.org/10.11646/ phytotaxa.314.2.2

Kumar, S., G. Stecher y K. Tamura. 2016. MEGA7: Molecular evolutionary genetics analysis version 7.0 for bigger datasets. Molecular Biology and Evolution 33(7): 1870-1874. DOI: https://doi.org/10.1093/molbev/msw054

Macler, B. A. 1988. Salinity effects on photosynthesis, carbon allocation, and nitrogen assimilation in the red alga, Gelidium coulteri. Plant Physiology 88 (3): 690-694. DOI: https://doi. org/10.1104/pp.88.3.690

Mateo-Cid, L. E. y A. C. Mendoza-González. 1991. Algas marinas bénticas de la Isla Cozumel, Quintana Roo, México. Acta Botanica Mexicana 16: 57-87. DOI: https://doi.org/10.21829/ abm16.1991.626

Mateo-Cid, L. E., A. C. Mendoza-González, A. G. Ávila-Ortiz, A. G. y S. Díaz Martínez. 2013. Algas marinas bentónicas del litoral de Campeche, México. Acta Botanica Mexicana 104: 53-92. DOI: https://doi.org/10.21829/abm104.2013.57

Mendoza-González, A. C y L. E. Mateo-Cid. 1992. Algas marinas bentónicas de Isla Mujeres, Quintana Roo, México. Acta Botanica Mexicana 19: 37-61. DOI: https://doi. org/10.21829/abm19.1992.646

Mendoza-González, A. C., L. E. Mateo-Cid y D. García-López. 2017. Inventory of benthic marine and estuarine algae and Cyanobacteria for Tabasco, Mexico. Biota Neotropica 17(4): e20170379. DOI: https://doi.org/10.1590/16760611-BN-2017-0379

Mendoza-González, A. C., L. E. Mateo-Cid y B. Searles. 2007. Yucatán seaweeds from the offshore waters of Isla Mujeres, Quintana Roo, Mexico. Botanica Marina 50(5): 280-287.

Núñez-Reséndiz, M. L. 2009. Catálogo de rodófitas del litoral del Morro de la Mancha, Veracruz. Tesis de licenciatura. Facultad de Ciencias, Universidad Nacional Autónoma de México. Cd. Mx., México. 145 pp.

Ortega, M. M. 1995. Observaciones del fitobentos de la Laguna de Términos, Campeche, México. Anales del Instituto de
Biología, Universidad Nacional Autónoma de México, Serie Botánica 66(1): 1-36.

Ortega, M. M., J. L. Godínez y G. Garduño-Solórzano. 2001. Catálogo de algas bénticas de las costas mexicanas del Golfo de México y Mar Caribe. Instituto de Biología, Universidad Nacional Autónoma de México. Cd. Mx., México. 594 pp.

Pacheco-Ruíz, I. y J. Zertuche-González. 1995. Effect of water movement on the growth of Gelidium robustum (Gardn.) Hollenb. and Abb. (Rhodophyta). Ciencias Marinas 21(1): 59-70. DOI: https://doi.org/10.7773/cm.v21i1.981

Perrone, C., A. Bottalico, G. H. Boo, S. M. Boo, K. A. Miller y D. W. Freshwater. 2019. Gelidium adriaticum sp. nov. and Gelidium carolinianum sp. nov. (Gelidiales, Rhodophyta) from the Mediterranean Sea. Phycologia 58(4): 359-373. DOI: https://doi.org/10.1080/00318884.2019.1580102

QGIS Development Team. 2009. QGIS Geographic Information System v. 2.18.28. Open Source Geospatial Foundation. http://qgis.org (consultado septiembre de 2021).

Quiroz-González, N., D. León-Álvarez y M. Rivas-Acuña. 2018. Biodiversidad de algas rojas marinas (Rhodophyta) en Tabasco, México. Acta Botanica Mexicana 123: 103-12. DOI: https://doi.org/10.21829/abm123.2018.1253

Quiroz-González, N., M. E. Ponce-Márquez, C. Fernández-García y D. Rodríguez. 2020. Gelidium gonzalezii sp. nov. (Gelidiales, Rhodophyta) from the Mexican tropical Pacific based on molecular and morphological evidence. Phytotaxa 459(2): 124-138. DOI: https://doi.org/10.11646/phytotaxa.459.2.4

Rambaut, A. 2010. FigTree v1.3.1. Institute of Evolutionary Biology, University of Edinburgh. Edinburgh, Scotland.

Ramírez, A. 1996. Estudio preliminar de las algas rojas (Rhodophyta) del litoral del Estado de Tabasco. Tesis de licenciatura. Universidad Nacional Autónoma de México, Campus Iztacala. Tlalnepantla, Estado de México, México. 66 pp.

Robledo, D., Y. Freile-Pelegrín e I. Sánchez-Rodríguez. 2003. Marine benthic algae from the Campeche Banks, México. In: Rosado-Espinoza, L., I. Ortegón-Aznar y M. Ruíz-Zárate (eds.). Proceedings of the XVII International Seaweed Symposium. Oxford University Press. Oxford, USA. Pp. 257-262.

Ronquist, F., M. Teslenko, P. van der Mark, D. Ayres, A. Darling, S. Höhna, B. Larget, L. Liu, M. A. Suchard y J. P. Huelsenbeck. 2012. MrBayes 3.2: Efficient Bayesian phylogenetic inference and model choice across a large model space. Systematic 
Biology 61(3): 539-542. DOI: https://doi.org/10.1093/sysbio/ sys029

Santelices, B. 1976. Taxonomic and nomenclatural notes on some Gelidiales (Rhodophyta). Phycologia 15(2): 165-173. DOI: https://doi.org/10.2216/i0031-8884-15-2-165.1

Santelices, B. 1988. Synopsis of biological data on the seaweed genera Gelidium and Pterocladia (Rhodophyta). FAO Fisheries Synopsis 145: 1-55.

Santelices, B. 1991. Production ecology of Gelidium. In: Juanes, J. A., B. Santelices, J. L. McLachlan (eds.). International Workshop on Gelidium. Developments in Hydrobiology, vol 68. Springer. Dordrecht, Netherlands. Pp.197-203. DOI: https://doi.org/10.1007/978-94-011-3610-5_3

Saunders, G. W. 2005. Applying DNA barcoding to red macroalgae: a preliminary appraisal holds promise for future applications. Philosophical transactions of the Royal Society B, Biological Sciences 360(1462): 1879-1888. DOI: https://doi.org/10.1098/rstb.2005.1719
Stamatakis, A. 2014. RAxML-VI-HPC: maximum likelihood-based phylogenetic analyses with thousands of taxa and mixed models. Bioinformatics 22(21): 2688-2690. DOI: https://doi. org/10.1093/bioinformatics/bt|446

Taylor, W. R. 1943. Marine algae from Haiti collected by H.H. Bartlett in 1941. Papers of the Michigan Academy of Sciences, Arts and Letters 28: 143-163.

Thompson, J. D., D. G. Higgins y T. J. Gibson. 1994. CLUSTAL W: improving the sensitivity of progressive multiple sequence alignment through sequence weighting, position-specific gap penalties and weight matrix choice. Nucleic Acids Research 22(22): 4673-4680. DOI: https://doi.org/10.1093/ nar/22.22.4673 